\title{
Simulated magnetic flows in the solar photosphere
}

\author{
S. Danilovic ${ }^{1}$, R. H. Cameron ${ }^{1}$, and S. K. Solanki ${ }^{1,2}$ \\ 1 Max-Planck-Institut für Sonnensystemforschung, Justus-von-Liebig-Weg 337077 Göttingen, Germany \\ e-mail: danilovic@mps.mpg.de \\ 2 School of Space Research, Kyung Hee University, Yougin, 446-701 Gyeonggi, Korea
}

Received 7 March 2014 / Accepted 12 August 2014

ABSTRACT

Context. Recent Sunrise/IMaX observations have revealed supersonic magnetic flows.

Aims. Our aim is to determine the origin of these flows by using realistic magnetohydrodynamics simulations.

Methods. We simulated cancellation and emergence of magnetic flux through the solar photosphere. Our first numerical experiment started with a magnetic field of both polarities. To simulate emergence into a region with pre-existing field, we introduced a large-scale horizontally uniform sheet of a horizontal field. We followed the subsequent evolution and created synthetic polarimetric observations, including known instrumental effects of the Sunrise/IMaX and Hinode/SP instruments. We compared the simulated and observed spectropolarimetric signals.

Results. Strongly blue- and redshifted Stokes $V$ signals are produced in locations where strong line-of-sight velocities coincide with the strong line-of-sight component of the magnetic field. The size and strength of simulated events is smaller than observed, and they are mostly associated with downflows, contrary to observations. In a few cases where they appear above a granule, single blue-lobed Stokes $V$ are produced by strong gradients in magnetic field and velocity. No change of magnetic field sign is detected along the line of sight in these instances. More high-speed magnetised flows occurred when an emergence was simulated than when no horizontal field was added.

Conclusions. The simulations indicate that the observed events result from magnetic flux emergences in which reconnection may take place, but does not seem to be necessary.

Key words. Sun: granulation - Sun: photosphere

\section{Introduction}

Advances in instrumentation often lead to the discovery of new phenomena in the solar atmosphere. Thus, Borrero et al. (2010) found a large number of strongly blue-shifted Stokes $V$ profiles in observations recorded by the Imaging Magnetograph eXperiment (IMaX, Martínez Pillet et al. 2011a) on board the Sunrise balloon-borne solar observatory (Barthol et al. 2011; Solanki et al. 2010; Berkefeld et al. 2011). By assuming an hG vertical field and after taking the IMaX spectral resolution into account, Borrero et al. (2012) concluded that supersonic velocities are needed to produce these signatures at a wavelength shift of $\Delta \lambda=+227 \mathrm{~m} \AA$ from the Fe I $525.02 \mathrm{~nm}$ nominal line centre. Since these features appear mostly above granules and thus are related to upflows, it is expected that the signal comes from the blue-shifted neighbouring Fe I $525.06 \mathrm{~nm}$ line.

Counterparts of these events were later found in Hinode/SP (Lites et al. 2001; Kosugi et al. 2007) data Martínez Pillet et al. (2011b). Martínez Pillet et al. (2011b) assumed that the Fe I $525.02 \mathrm{~nm}$ and Fe I $630.25 \mathrm{~nm}$ lines are similar and equally sensitive to all atmospheric parameters and identified signals that correspond to the same Doppler velocities. The difference with respect to IMaX results is that while IMaX found $70 \%$ of the cases to be associated with upflows, in Hinode data blue-shifted and red-shifted profiles were equally present. On the other hand, both datasets as well as the detailed study by Quintero Noda et al. (2013) showed that the events coincide with the appearance of linear polarisation in the regions where the sign of

* The movies are available in electronic form at http://www. aanda.org the line-of-sight field was mixed. Based on these characteristics, both groups of authors concluded that reconnection of the emerging with the pre-existing field might be the cause of these magnetic flows. This was supported by Borrero et al. (2013), who found a reversal in the polarity of the magnetic field along the line of sight, accompanied by an enhancement in the temperature and supersonic line-of-sight velocities.

We explore this scenario by using numerical 3D radiation magnetohydrodynamics (MHD) simulations of an emerging magnetic flux sheet in the presence of magnetic flux concentrations. We compute spectral line profiles in the simulated snapshots to simulate the IMaX or Hinode observations and compare the results with the observed signatures.

\section{Computational runs and synthetic observations}

We considered two runs produced with the code MURaM (Vögler et al. 2005). The setup is essentially the same as in Cameron et al. (2011), with a checkerboard-like $2 \times 2$ pattern of initial magnetic polarity and a vertical magnetic field boundary condition at the upper boundary that is transparent for upflows and non-grey radiative transfer included. The computational domain of $6 \times 6 \times 1.68 \mathrm{Mm}$ was chosen with some $700 \mathrm{~km}$ above $\tau_{500}=1$ and a resolution of $10 \mathrm{~km}$ and $14 \mathrm{~km}$ in horizontal and vertical directions. Both ambipolar diffusion and the Hall current were included in the induction equation. These terms are expected to be important in the photosphere for dynamics at very small scales, especially in regions where the magnetic field gradients are large (Cheung \& Cameron 2012). At the current resolution (10 km in the horizontal directions) the effects on 

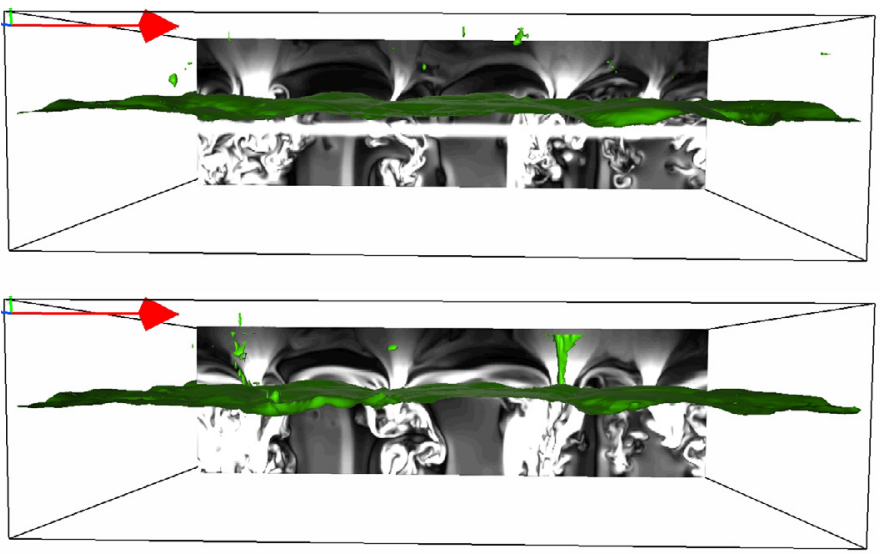

Fig. 1. Two snapshots of the emergence run - side view of the simulation domain at $t=0 \mathrm{~s}$ (upper) and $t=188 \mathrm{~s}$. The vertical plane shows the magnetic field strength. The direction of the introduced horizontal field is shown with red arrows ( $\mathrm{X}$ direction). The green isosurface of a temperature of $6000 \mathrm{~K}$ marks the position of the $\tau_{500} \approx 1$ level. Above this corrugated plane, green features are visible that signal hot gas in the upper part of the simulation domain. This is where reconnection takes place or shock fronts are formed.

the dynamics of quiet-Sun features are relatively weak (Cheung \& Cameron 2012). A study of the full effects of the ambipolar and Hall effects is an interesting topic for future studies at higher spatial resolution.

Both runs started from the same snapshot of non-magnetic convection, to which a purely vertical magnetic field of positive and negative polarity was added. This initial field was arranged in a $2 \times 2$ checkerboard pattern (as in Cameron et al. 2011) and had a height-independent strength of $200 \mathrm{G}$. The first run (the reference run) was allowed to evolve freely from this initial condition, simulating the decay of the magnetic field in a mixedpolarity region. For the second run we introduced a horizontal flux sheet $300 \mathrm{~km}$ below the mean optical depth unity to simulate the scenario explained in the introduction. The field strength was set to vary across the cross-section as a Gaussian with a full width at half maximum (FWHM) of $110 \mathrm{~km}$ with a maximum value of $1 \mathrm{kG}$. We call this run the emergence run. All times are given throughout with respect to the time at which the flux sheet was introduced in the second run.

Figure 1 shows a vertical cut through the initial snapshot (with the imposed sheet of horizontal flux) and the snapshot $188 \mathrm{~s}$ later. In the upper panel, the sheet is unmodulated and in its initial position. The field strength of $1 \mathrm{kG}$ at the centre of the sheet gradually decrease to $0 \mathrm{G}$ at the edges. The added field was directed in the $+X$ axis, that is, it is aligned with the planes shown in this figure. At $t=188 \mathrm{~s}$ most of the flux in this sheet has emerged. At this time, high-temperature structures are visible in locations where the emerging flux reconnects with the pre-existing magnetic field (green features in the upper part of the simulation domain). We stress that it is not our aim to follow the detailed emergence and evolution of individual loops or the sheet in general. Instead, we are interested in the interaction of the emerging flux with the pre-exiting field in the solar photosphere. The number of these interactions is maximised with the initial magnetic configuration chosen here. Additionally, the time needed for the field to emerge is minimised.

To explore the observational signatures of these interactions, we synthesised the Sunrise/IMaX and Hinode/SP observables with the radiative transfer code SPINOR (Frutiger et al. 2000), which is based on the STOPRO routines (Solanki 1987). The
Fe abundance used for the synthesis is taken from Thevenin (1989) and the values of the oscillator strengths from the VALD database (Piskunov et al. 1995). For simulated IMaX data we synthesised the Fe I $525.02 \mathrm{~nm}$ line, the Fe I $525.06 \mathrm{~nm}$ line, and the Co I $525.0 \mathrm{~nm}$ line, which were then smeared with the IMaX spectral point spread function (PSF). To simulate the effect of the phase diversity reconstruction technique with which the data were reconstructed that Borrero et al. (2010) used, we applied a low-pass filter that removes all spatial frequencies higher than $5.5 \operatorname{arcsec}^{-1}$. We also took into account the straylight, which was estimated empirically based on data recorded at the limb (Feller et al., in prep.). A noise of $3 \times 10^{-3} I_{\mathrm{c}}$ was added.

The Hinode/SP lines Fe I 630.15 and $630.25 \mathrm{~nm}$ were also computed. They were broadened with the Hinode/SP spectral PSF and with a realistic spatial PSF (Danilovic et al. 2008). Finally, a random noise of $8 \times 10^{-4} I_{\mathrm{c}}$ was added.

\section{Results}

The simulation without the imposed horizontal flux sheet is similar to that studied by Danilovic et al. (2010). The main dynamical activity is the cancellation of flux between the opposite-polarity magnetic elements. High velocities only occur in a few locations where several granules meet and magnetic field intensification takes place. Since the addition of the horizontal flux sheet leads to a substantial increase in the number of high-velocity events, we concentrate mainly on the emergence run. Figure 2 shows various plasma properties on the $\left\langle\tau_{500}\right\rangle=1$ plane from this simulation at $t=156 \mathrm{~s}^{1}$.

The flux sheet is significantly corrugated by the convective flows already $30 \mathrm{~s}$ after the start. At around $90 \mathrm{~s}$, the first horizontal field crosses the $\left\langle\tau_{500}\right\rangle=1$ plane. Then at $156 \mathrm{~s}$ the whole simulation domain at that level is covered with horizontal magnetic field structures. Although the field strength was initially uniform in the horizontal direction, the loops now are highly structured. Fine structures in the magnetic field are reflected in the fine structures in intensity. The vertical field map shows footpoints of $\Omega$ loops as they sweep from the centres of granules towards their edges, at the same time increasing in strength as the material drains.

As the field emerges, strong upflows appear at the $+\mathrm{X}$ edge of the pre-existing magnetic elements with negative polarity and on the $-\mathrm{X}$ edge of positive magnetic elements. These are locations at which the emerging field has the opposite polarity to the pre-existing field. An examination of the magnetic field lines shows that the high velocities correspond to U loops that are formed by reconnection below the surface and are accelerated outwards by magnetic tension as they cross the photosphere. This outward acceleration leads to upflows and corresponding blue-shifts of the spectral lines. In some cases the reconnection occurs at optical depth unity where strong magnetic concentrations are pushed together by the granular flow. Reconnection jets with both upflows and downflows are then present ${ }^{2}$. Similar jets have previously been studied in more idealised simulations at different spatial scales (Takeuchi \& Shibata 2001; Isobe et al. 2008; Kigure et al. 2010).

On the other side of the flux concentrations (the $-\mathrm{X}$ edge of the features with negative polarity and $+X$ side of the

\footnotetext{
1 Movies covering the 20-min period of both runs are available (online). They combine all the maps shown in Figs. 2-4.

2 An example of these processes can clearly be seen in the accompanying movie around $t=406 \mathrm{~s}$ at $[2.5,4.5] \mathrm{Mm}$.
} 

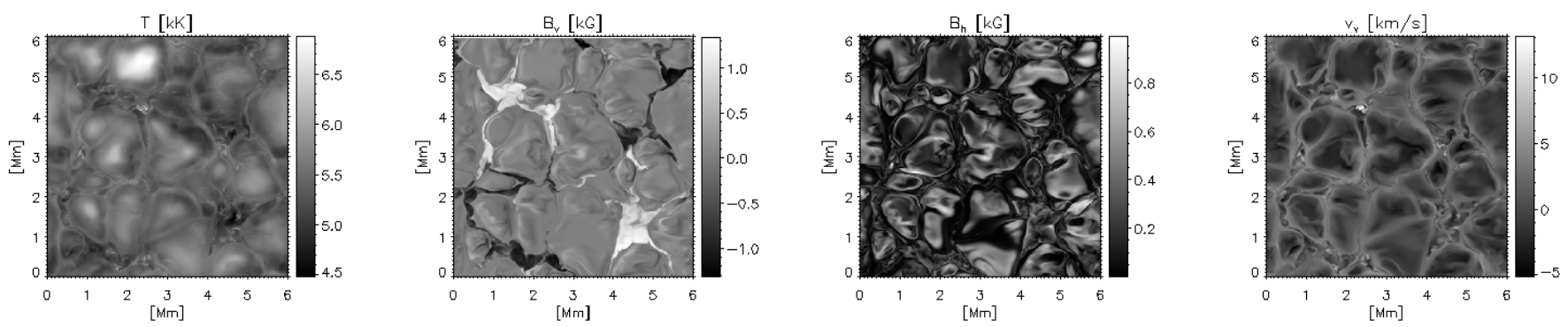

Fig. 2. Emergence run at $t=156 \mathrm{~s}$ - horizontal cut through the simulation domain (for the evolution of these quantities - see the online movie). Left to right: temperature, vertical, and horizontal field, line-of-sight velocity (upflow is negative) at a constant geometrical height that corresponds to $\left\langle\tau_{500}\right\rangle \approx 1$.
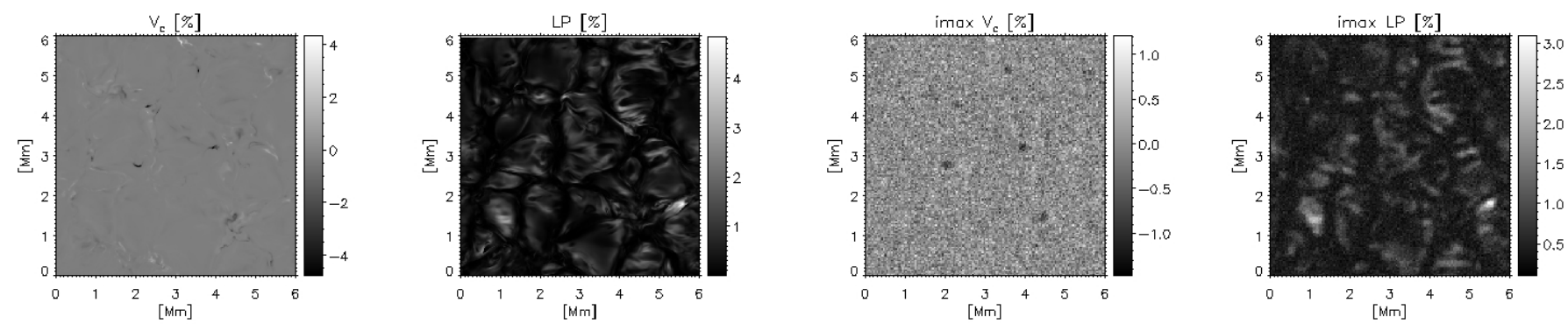

Fig. 3. Synthesised Sunrise/IMaX observables from the emergence run at $t=156 \mathrm{~s}$ (for the evolution of these quantities - see the online movie). Left to right: Stokes $V \Delta \lambda=+227 \mathrm{~m} \AA$ away from the nominal Fe I $525.02 \mathrm{~nm}$ line centre and mean linear polarisation at the original and the IMaX resolution.
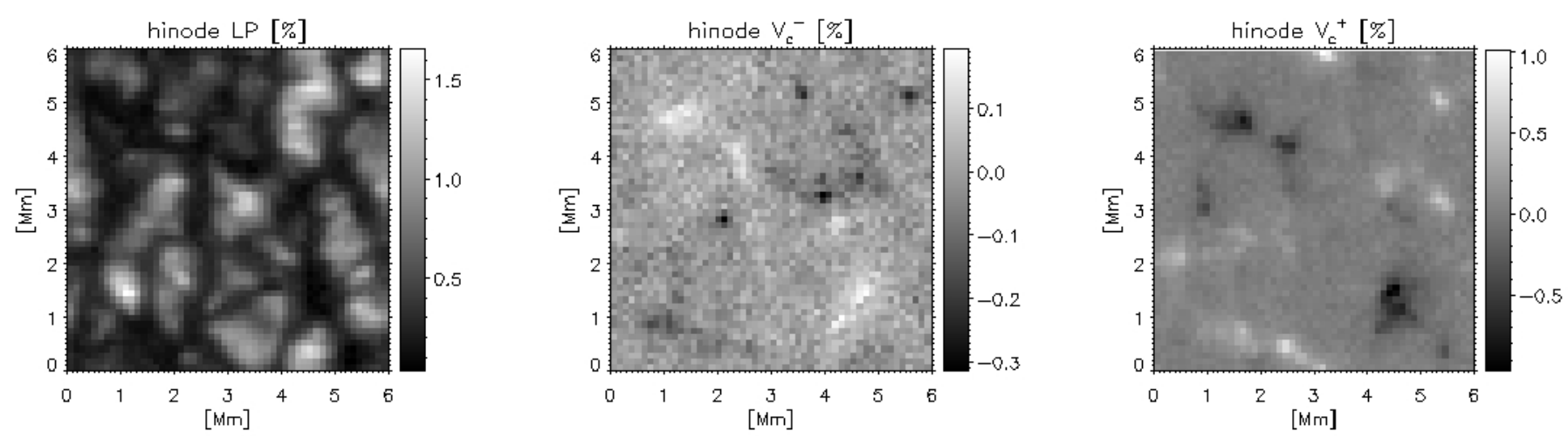

Fig. 4. Synthesised Hinode/SP observables from the emergence run at $t=156 \mathrm{~s}$ (for the evolution of these quantities - see the online movie). Left to right: mean linear polarisation and Stokes $V$ at $\Delta \lambda=-227 \mathrm{~m} \AA$ and $+227 \mathrm{~m} \AA$ away from the nominal Fe I $630.25 \mathrm{~nm}$ line centre.

positive-polarity features), the emerging flux does not lead to a cancellation, but to an enhancement of the field. This is consistent with the fact that the horizontal flux sheet has zero net vertical flux. This enhancement of the magnetic field can initiate a "collapse" of the field (old and new) to kilo-gauss strengths. This process is aided in some cases by the draining of material down the field lines and produces strong negative vertical velocities, and consequently, strong red-shifted features ${ }^{3}$.

\subsection{Sunrise/IMaX observables}

We now consider the resulting spectropolarimetric signals as they would be seen by IMaX (Fig. 3). A parameter $V_{\mathrm{c}}$ is defined here as a Stokes $V$ signal at $\Delta \lambda=+227 \mathrm{~m} \AA$ from the Fe I $525.02 \mathrm{~nm}$ nominal line centre. Without the imposed flux

\footnotetext{
3 An examples of these processes can clearly be seen in the accompanying movie around $t=375 \mathrm{~s}$ at $[2.3,2.8] \mathrm{Mm}$.
}

sheet, there are only a few events where significantly strong $V_{\mathrm{c}}$ appear. The number of these events increases significantly for the emergence run, with the highest frequency of the events appearing in the period from $t=62 \mathrm{~s}$ to $t=375 \mathrm{~s}$ during which most of the horizontal field appears at the surface. The strong $V_{\mathrm{c}}$ signals appear mostly in intergranular lanes. The locations of the strongly shifted signals change slightly as the granular motions buffet the magnetic features. The drift velocities are comparable with observations, as reported by Borrero et al. (2010).

The simulated $V_{\mathrm{c}}$ features sometimes appear in pairs, for instance the example at $t=313 \mathrm{~s}$ at $[2.4,3] \mathrm{Mm}$ in the accompanying movie. The same is seen in observations, where these pairs of high $V_{\mathrm{c}}$ signals coincide with locations where the mass drains and strong downflows are generated. At the same time, only very weak $V_{\mathrm{c}}$ signals are present where upflows arise, especially after spatial smearing. Figure 5 displays this asymmetry between up- and downflow. We note that in general only $V_{c}>5 \%$ at the original spatial resolution of the simulations remains above 

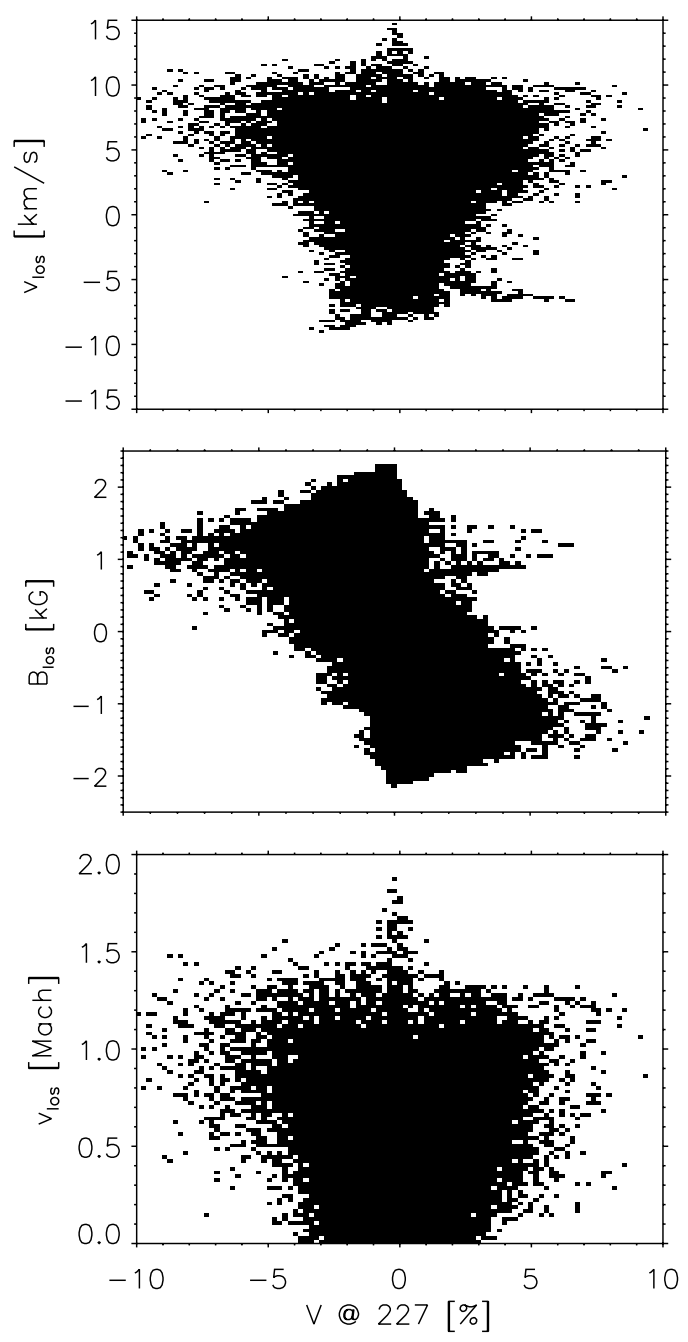

Fig. 5. Scatter plots of the synthesised IMaX Stokes $V$ signal at $\Delta \lambda=$ $227 \mathrm{~m} \AA$ from Fe I 525.02 nm nominal line centre (i.e. "continuum" Stokes $V$ or $V_{\mathrm{c}}^{-}$) versus line-of-sight velocity (top panel) and magnetic field (middle panel) at $\log \tau=-1$. Bottom panel: the line-of-sight velocity in units of the Mach number.

the detection threshold after the spatial smearing. $V_{\mathrm{c}}$ with this strength can only be created in locations where the vertical component of the magnetic field exceeds $1 \mathrm{kG}$ combined with velocities higher than $5 \mathrm{~km} \mathrm{~s}^{-1}$. The upflows that appear in the intergranular lanes coincide with strongly bent magnetic field lines, so that in these cases the vertical component of the magnetic field is rather weak. This explains why the scatter plot in Fig. 5 (middle panel) shows that almost all $V_{\mathrm{c}}>5 \%$ are associated with downflows. The bottom panel of Fig. 5 reveals that only $<1 \%$ of pixels contains supersonic velocities. A very low percentage of these also shows $V_{\mathrm{c}}>5 \%$, and they are all observed in downflows.

To study the statistics of these events that show high $V_{\mathrm{c}}$ signals, we used the threshold of $\left|V_{\mathrm{c}}\right|>1.25 \times 10^{-2} I_{\mathrm{c}}$ on the smeared $V_{\mathrm{c}}$ maps, corresponding to $\approx 4 \sigma$, which is the same threshold as used by Borrero et al. (2010). Instead of using the minimum of nine IMaX pixels, we took three as a minimum size of the events. In this way, we detected three cases in the reference run and 23 cases in the emergence run. Figure 6 shows characteristics of these events. The comparison with Fig. 2 from Borrero et al. (2010), which shows the corresponding histograms from the observations, reveals several interesting facts. Firstly, the
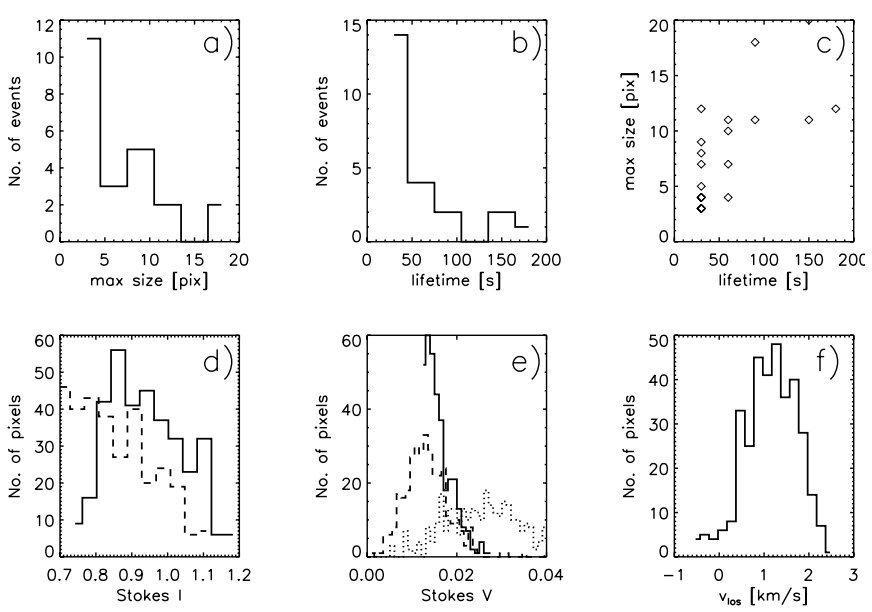

Fig. 6. Characteristics of selected simulated events: a) distribution of maximal area, b) lifetimes, c) relations between the area and the lifetime, d) corresponding Stokes I profiles (solid - continuum intensity distribution; dashed - line averaged intensity), e) corresponding Stokes $V$ profiles (solid - Stokes $V$ at $\Delta \lambda=+227 \mathrm{~m} \AA$; dashed - mean, line-averaged Stokes $V$; dotted - maximum of Stokes $V$ ) and f) line of sight velocity retrieved from Stokes I. The figure is comparable to Fig. 2 from Borrero et al. (2010) which shows the same parameters retrieved from observations.

simulated events are smaller and shorter lived than the observed events (Figs. 6a and b). This is to be expected given the lower size threshold used here. Although there are too few events to be certain, there is a hint that the correlation between size and lifetime also exists in simulations (Fig. 6c). Thirdly, the continuum intensities in these regions are slightly lower than in observation (Fig. 6d). This is understandable because most of the events occur in the intergranular lanes. The line-averaged circular polarisation signal (as calculated by Borrero et al. 2010) is lower than the corresponding $V_{\mathrm{c}}$ (Fig. 6e). However, the Stokes $V$ profiles are mostly normal with an extended red lobe or additional third red-shifted lobe. Instead, if we also overplot the highest amplitude of the simulated Stokes $V$ profiles, we find the behaviour we expected, which is that the strongest signals are mostly not at $\Delta \lambda=+227 \mathrm{~m} \AA$ away from the IMaX line. It seems therefore that averaging over four wavelength points $\Delta \lambda=[-80,-40,40,80] \mathrm{m} \AA$ is a poor diagnostics, since it gives a false image of the $V$ profiles in question. Finally, the most conspicuous difference with respect to observations is visible in the line-of-sight velocity histogram (Fig. 6e). While most observed events are associated with upflows, as deduced from the Stokes $I$ profiles, the vast majority of the simulated events is associated with downflows. In the few exceptions, upflows of no more than $500 \mathrm{~m} / \mathrm{s}$ are detected.

Two of the simulated cases associated with upflows are shown in Fig. 3. The atmospheric parameters for the case at location [2,2.8] $\mathrm{Mm}$ are given in Fig. 7, the resulting line profiles in Fig. 8. The other example at $[4,3.4] \mathrm{Mm}$ is almost the same. In both cases, the new flux emerges, and where the material is more magnetised, hence more buoyant, the combination of strong upflows and the magnetopause produces single-lobed Stokes $V$ profiles with only blue lobes (Steiner 2000; Sainz Dalda et al. 2012). The maps in the upper row in Fig. 7 show that the strong $V_{\mathrm{c}}$ signal coincides with a strip of material that carries a field of $1 \mathrm{kG}$ and has an upflow velocity of $>7 \mathrm{~km} \mathrm{~s}^{-1}$. The height profiles (lower row in Fig. 7) show that both magnetic field and velocity rapidly decrease with 

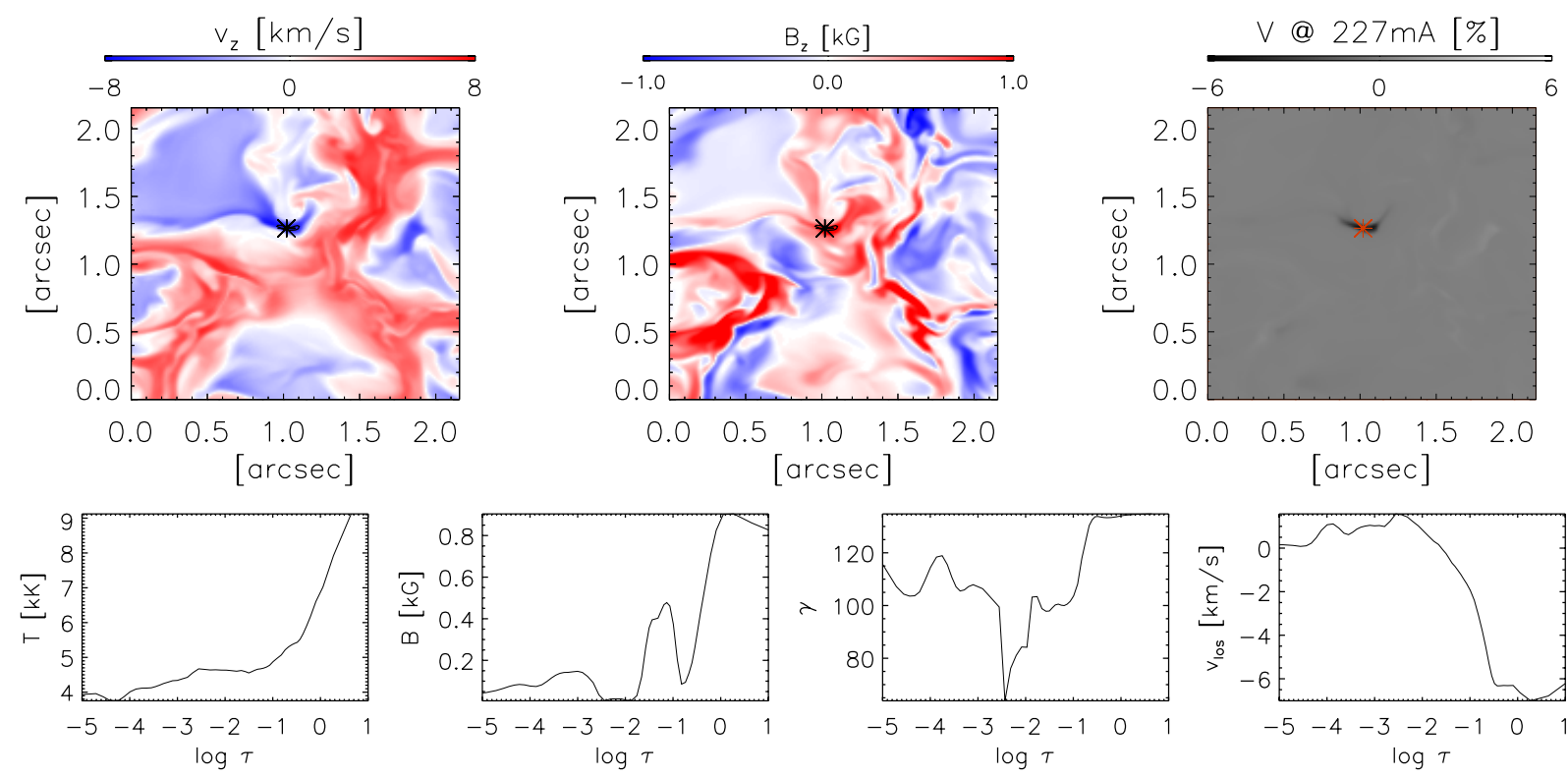

Fig. 7. An example of a feature that appears on the edge of a granule in the "emergence run" at $t=156 \mathrm{~s}$. The feature is visible at [2,2.8] Mm in Fig. 3. Upper row, left to right: line-of-sight (LOS) velocity, and vertical magnetic field at $\log \tau=-1$ and corresponding map of Stokes $V$ at $\Delta \lambda=+227 \mathrm{~m} \AA$ at original spatial resolution. The star symbols mark the position of the pixel of interest. Lower row, left to right: height profiles of temperature, magnetic field strength, inclinations and LOS velocity at the pixel of interest.

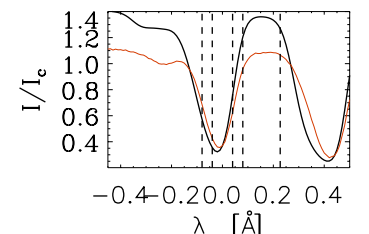

$\lambda \quad[\AA]$
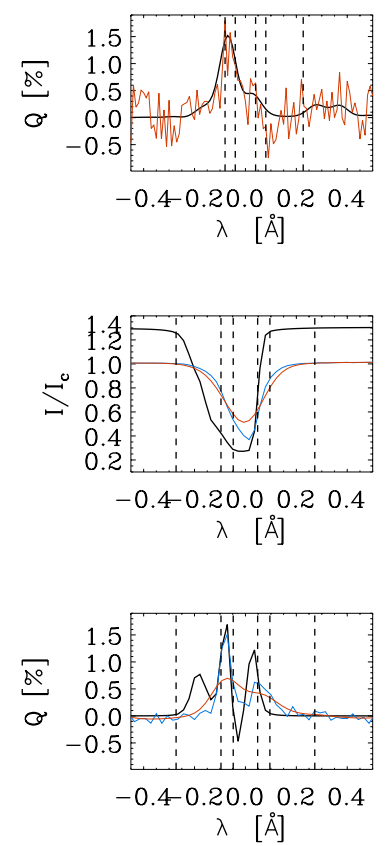

Fig. 8. Example of a feature that appears on a granular edge in the emergence run at $t=156 \mathrm{~s}$. Resulting Stokes $I, Q, U$ and $V$ profiles at the pixel shown in Fig. 7. Upper panels: synthesised IMaX observations at the original resolution (black line) and for the diffraction-limited resolution, stray-light and noise are taken into account (red line); lower panels: synthesised Hinode observations, the Fe I $630.25 \mathrm{~nm}$ at the original spatial resolution (black), at the spatial resolution of Hinode (blue), and with the spectral resolution of IMaX (red). height, which means that these types of events will appear at the moment when the newly emerging flux reaches the solar surface. The resulting profile remains single-lobed with the signal higher than the detection limit (Fig. 8). In this case, the linear polarisation signal also remains above the noise level.

\subsection{Hinode/SP observables}

To determine whether these events are visible with Hinode/SP, we followed Martínez Pillet et al. (2011b). Synthesised Hinode/SP observables were additionally spectrally smeared with a Gaussian with a FWHM of $95 \mathrm{~m} \AA$, and the three maps shown in Fig. 4 were produced. The mean linear polarisation maps were obtained by averaging the linear polarization signal over four wavelength points $[-96,-48,48,96] \mathrm{m} \AA$ away from the Fe I $630.25 \mathrm{~nm}$ line centre. The maps are somewhat different from IMaX counterparts because the IMaX line is formed higher up and tends to sample higher loops (Danilovic et al., in prep.), which combined with the higher spatial resolution of IMaX gives them a stripe-like shape. Moreover, different noise levels are important factors because most of the weak signals in the case of IMaX are lost. To identify up- and downflow events, as in Martínez Pillet et al. (2011b), the circular polarization maps $V_{\mathrm{c}}^{-}$ and $V_{\mathrm{c}}^{+}$were taken at $\mp 272 \mathrm{~m} \AA$. Because of the different widths of the Fe I $525.02 \mathrm{~nm}$ and Fe I $630.25 \mathrm{~nm}$ lines and their different sensitivity to velocities (as well as different noise levels), the strong magnetic features are prominent in the $V_{\mathrm{c}}^{-}$and $V_{\mathrm{c}}^{+}$maps. Martínez Pillet et al. (2011b) found the same in observations and called it "leakage from magnetic network".

The emergence run movie shows that most of the events seen in simulated IMaX observations are also visible in $V_{\mathrm{c}}^{+}$maps, as expected. These profiles are very asymmetric with an extended red wing or an additional red lobe. On the other hand, only few events are visible in $V_{\mathrm{c}}^{-}$. Two events appear above the granule at $t=156 \mathrm{~s}$ and are exactly at the detection limit if a threshold of $0.32 \%$ is employed, as chosen by Martínez Pillet et al. (2011b). The lower panels in Fig. 8 show the corresponding profiles. At 
the original spatial resolution, the Stokes $V$ profile is singledlobed with an amplitude of $>15 \%$. After the spatial smearing this becomes a three-lobed profile with a blue-shifted third lobe. The asymmetry in Stokes $I$ completely vanishes after the spectral smearing, and, as for IMaX, Stokes $Q$ remains above the noise.

\section{Conclusions}

Recent Sunrise/IMaX observations revealed many short-lived events during which highly shifted circular polarization signals were detected. Their apparent association with the appearance of a linear polarization signal and the nearby opposite magnetic polarity suggest that these events might be produced by the reconnection of the emerging with the preexisting field. Here, we used realistic 3D MHD simulations to investigate the applicability of this scenario. We introduced a uniform horizontal field $\approx 300 \mathrm{~km}$ below the solar surface into a mixed-polarity run and searched for counterparts to the observed signatures. We simulated Sunrise/IMaX and Hinode/SP observables. The simulations showed that events similar to the observations tend to appear much more often when emergence is taking place and only in the locations where the high line-of-sight velocities coincide with the strong longitudinal magnetic field. These simulated events qualitatively match the observations. The synthesised line profiles are very similar to the observed ones, but there are a few significant differences. The simulated events are on average considerably smaller and weaker than observed. For the mean lifetimes and sizes of these events we obtained $57.4 \mathrm{~s}$ and 7.6 pixels, instead of $81.3 \mathrm{~s}$ and 15.5 pixels, as found by Borrero et al. (2010) in the observations. Moreover, the huge majority of them appears in intergranular lanes and is associated with downflows, in contrast to observations. The size limit introduced by Borrero et al. (2010) probably removed most of these events. As a result, their analysis included only the cases that appear above a granule. We found a few of them in our simulations. They are produced at the moment when the newly emerging flux appears at the surface. At these locations, magnetic field and velocity rapidly decrease with height. We found no change of polarity along the line of sight, as suggested by Borrero et al. (2013). In addition, our simulation showed none of the height profiles presented in that paper. In the simulations, reconnection occurs in the intergranular lanes where magnetic features of opposite polarity meet. Depending on the gradient of magnetic field, the temperature height profile can show a bump corresponding to an increase of $\approx 1000 \mathrm{~K}$. As a result, a shift in the formation height of the synthesised Fe I lines is produced. Because of this, lines usually do not reveal dramatic events or show only associated downflows that occur below the temperature bump, that is, below the reconnection site.
Why did we not produce more examples that matched the observations? Considering that these features appear very seldom, only a few over 55" × 55" (Martínez Pillet et al. 2011b), this is not surprising. This means that we need simulations that cover larger field of view and extend deeper into the convention zone. We expect that such simulations will result in more realistic emergence events and wider spectra of magnetic field configurations that are not imposed by initial and boundary conditions, as in our case.

\section{References}

Barthol, P., Gandorfer, A., Solanki, S. K., et al. 2011, Sol. Phys., 268, 1 Berkefeld, T., Schmidt, W., Soltau, D., et al. 2011, Sol. Phys., 268, 103 Borrero, J. M., Martínez-Pillet, V., Schlichenmaier, R., et al. 2010, ApJ, 723, L144

Borrero, J. M., Pillet, V. M., Schlichenmaier, R., et al. 2012, 4th Hinode Science Meeting: unsolved problems and recents insights, eds. L. R. Bellot Rubio, F. Reale, \& M. Carlsson (San Francisco: ASP), ASP Conf. Ser., 455, 155 Borrero, J. M., Martínez Pillet, V., Schmidt, W., et al. 2013, ApJ, 768, 69 Cameron, R., Vögler, A., \& Schüssler, M. 2011, A\&A, 533, A86 Cheung, M. C. M., \& Cameron, R. H. 2012, ApJ, 750, 6

Danilovic, S., Gandorfer, A., Lagg, A., et al. 2008, A\&A, 484, L17

Danilovic, S., Schüssler, M., \& Solanki, S. K. 2010, A\&A, 509, A76

Frutiger, C., Solanki, S. K., Fligge, M., \& Bruls, J. H. M. J. 2000, A\&A, 358, 1109

Isobe, H., Proctor, M. R. E. \& Weiss, N. O. 2008, ApJ, 679, L57

Kigure, H. Takahashi, K., \& Shibata, K. et al. 2010, PASJ, 62, 993

Kosugi, T., Matsuzaki, K., Sakao, T., et al. 2007, Sol. Phys., 243, 3

Lites, B. W., Elmore, D. F., Streander, K. V., et al. 2001, Proc. SPIE, 4498, 73

Martínez Pillet, V., Del Toro Iniesta, J. C., Álvarez-Herrero, A., et al. 2011 Sol. Phys., 268, 57

Martínez Pillet, V., Del Toro Iniesta, J. C., \& Quintero Noda, C. 2011, A\&A, 530, A111

Piskunov, N. E., Kupka, F., Ryabchikova, T. A., Weiss, W. W., \& Jeffery, C. S. 1995, A\&AS, 112, 525

Quintero Noda, C., Martínez Pillet, V., Borrero, J. M., \& Solanki, S. K. 2013, A\&A, 558, A30

Sainz Dalda, A., Martínez-Sykora, J., Bellot Rubio, L., \& Title, A. 2012, ApJ, 748,38

Solanki, S. K. 1987, Ph.D. Thesis, Zurich

Solanki, S. K., Barthol, P., Danilovic, S., et al. 2010, ApJ, 723, L127

Steiner, O. 2000, Sol. Phys., 196, 245

Takeuchi, A., \& Shibata, K. 2001, ApJ, 546, L73

Thevenin, F. 1989, A\&AS, 77, 137

Vögler, A., Shelyag, S., Schüssler, M., et al. 2005, A\&A, 429, 335

\section{Appendix A}

Two movies for the reference and the emergence runs are attached to the paper. Figures A.1 and A.2 show the styles from the movies. 
S. Danilovic et al.: Simulated magnetic flows
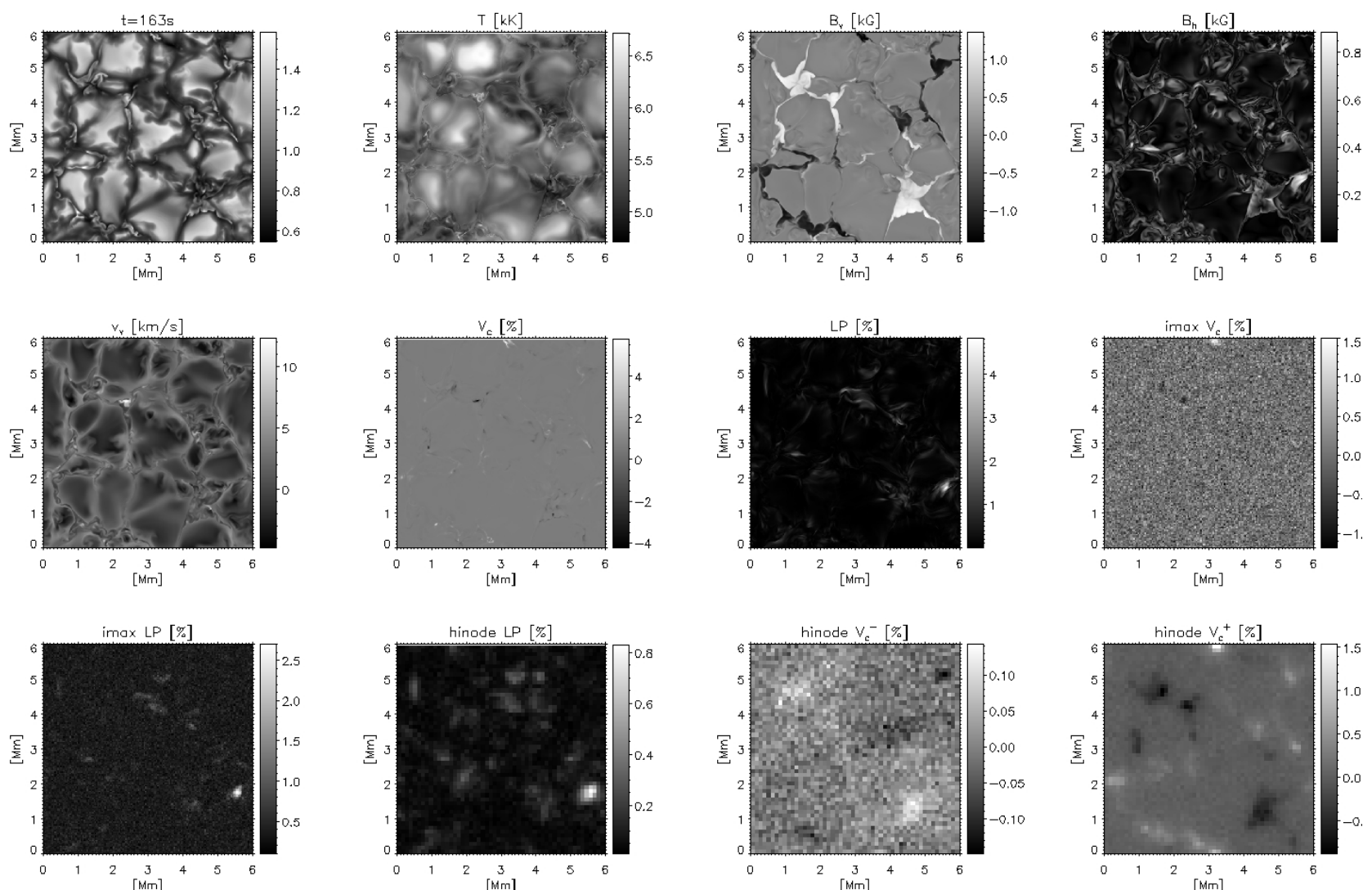

Fig. A.1. Snapshot from the reference run. From left to the right and from top to the bottom: bolometric intensity; temperature, vertical and horizontal field, line-of-sight velocity (upflow is negative) at constant geometrical height that corresponds to $\left\langle\tau_{500}\right\rangle \approx 1 ;$ Stokes $V \Delta \lambda=+227 \mathrm{~m} \AA$ away from the nominal Fe I $525.02 \mathrm{~nm}$ line centre and mean linear polarisation at original and IMaX resolution; mean linear polarisation and Stokes $V$ at $\Delta \lambda=-227 \mathrm{~m} \AA$ and $+227 \mathrm{~m} \AA$ away from the nominal Fe I $630.25 \mathrm{~nm}$ line centre.
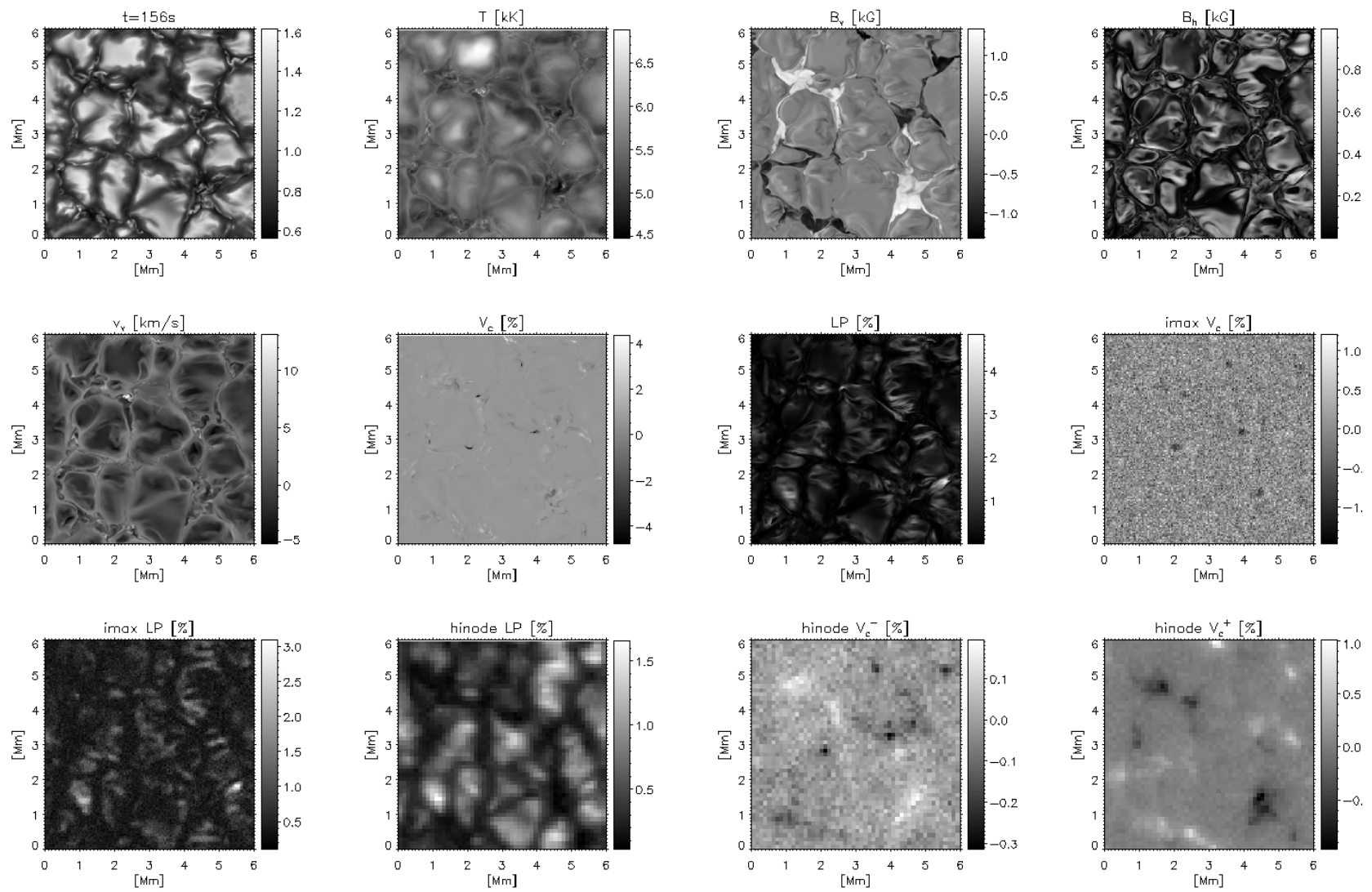

Fig. A.2. Snapshot from the emergence run. The same as in Fig. A.1. 